\title{
Integration of Automated Decision Support Systems with Data Mining Abstract: A Client Perspective
}

\author{
Abdullah Saad AL-Malaise \\ Chairman of Information Systems Department \\ Faculty of Computing and Information Technology \\ King Abdulaziz University, Jeddah \\ Kingdom of Saudi Arabia.
}

\begin{abstract}
Customer's behavior and satisfaction are always play important role to increase organization's growth and market value. Customers are on top priority for the growing organization to build up their businesses. In this paper presents the architecture of Decision Support Systems (DSS) in connection to deal with the customer's enquiries and requests. Main purpose behind the proposed model is to enhance the customer's satisfaction and behavior using DSS. We proposed model by extension in traditional DSS concepts with integration of Data Mining (DM) abstract. The model presented in this paper shows the comprehensive architecture to work on the customer requests using DSS and knowledge management (KM) for improving the customer's behavior and satisfaction. Furthermore, DM abstract provides more methods and techniques; to understand the contacted customer's data, to classify the replied answers in number of classes, and to generate association between the same type of queries, and finally to maintain the KM for future correspondence.
\end{abstract}

Keywords-Decision Support Systems; Knowledge Management; Data Mining; Customer's Satisfaction.

\section{INTRODUCTION}

Customers are regularly in contact with the organizations through telephone lines, online website portal, or through customer care centers directly. The organizations keen to learn their employees about how to deal with the customers in efficient manner. Customer's satisfaction and to improve in their behavior with the company is the main aim for every organization. As customers are supposed large stakeholders; those can increase and decrease the profit ratio of the company.

Abdullah et al. [9] described that customer's feedback and satisfaction level is the major performance indicators to improve customer's behavior which a customer leaves behind of his/her every visit to the company. In this paper we presented the model of automated decision support systems for dealing with the customer's queries for increase the customer's satisfaction ration. For this, we integrated the model with DM abstract to build a KM database in connection with automated DSS database. Ruey [3] discussed the connection of DM technique with customer's data that, the systemic applications of DM strengthens the KM process and allows marketing personnel to know their customers well to provide better services. Figure. 1 by [13] showed the working of traditional hot-line customer service center which representing the long process of customer dealing via telephone line and advisory system. Which shows that in current days we cannot relied upon dealing with the customers in traditional manner. There is a need to focus on current trends and technology to improve the performance and growth of the organization.

Before discussing the proposed model in this paper, the subsequent sections discussing the introduction of DSS and DM approaches using literature are reviewed to understand more about the subject matter. Therefore, in the next section the discussion about the DSS configuration and components is presented. Followed by discussion on DM tasks to provide some background of it too.

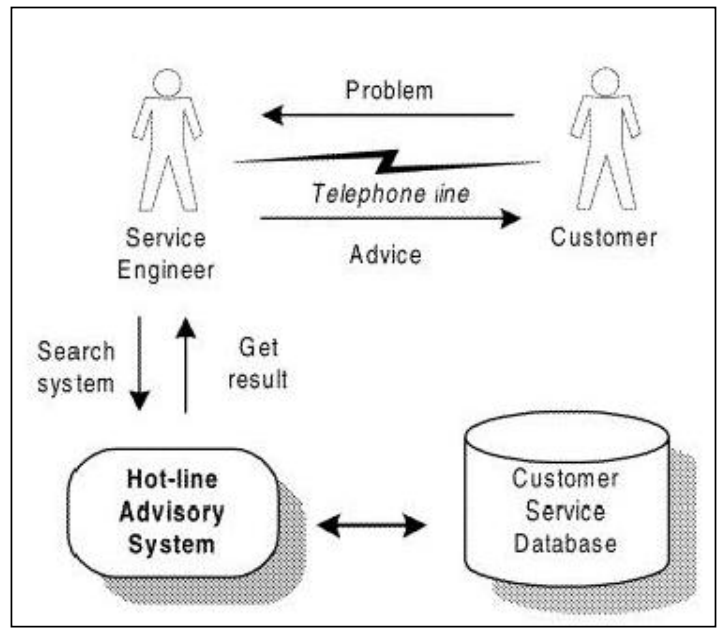

Fig. 1. Traditional Hot-Line Service Center [13]

\section{A. Decision Support System}

Currently, business environment is more complex than ever before. The reason behind is the rapid growth and involvement of the technology in the businesses. Modern technology is managing the cause of increasing business pressure on the business men. Furthermore, the businesses are need to be updated using current technology to defend their selves from competitor's attack timely. The computerized support and automated decision support systems are becoming the fundamental elements for almost every organization to stay in the market safe and healthy.

DSS provides the environment to apply many tools for all kind of business environmental factors such as; markets, technology, customer demand, and societal \& environmental 
factors to generate several choices and in the end to be selected optimal solution [2]. A DSS can have several phases to analyze the problem and take the decision. As Turban described a DSS must consider four phases for complete decision making process such as; Intelligent, Design, Choice and Implementation [2].

Industries build DSS for making themselves proactive and anticipative. The configuration of DSS can be based on data or model. The major classification of DSS are based on (i) Data Oriented DSS, (ii) Model Oriented DSS [2]. While some other scholars also categorized DSS into several categories such as; individual and group DSS, spreadsheet DSS, solver oriented DSS, etc.

Figure-2 [2] showed the basic model of DSS including linkages with its components. In this figure showing the four parts of DSS are; data, models, knowledge and user interface. In this paper we are applying the same concept with some integration in the model, discussed later in methodology section.

In the succeeding section presented the most common DM approaches and tasks which will describe more about the DM background.

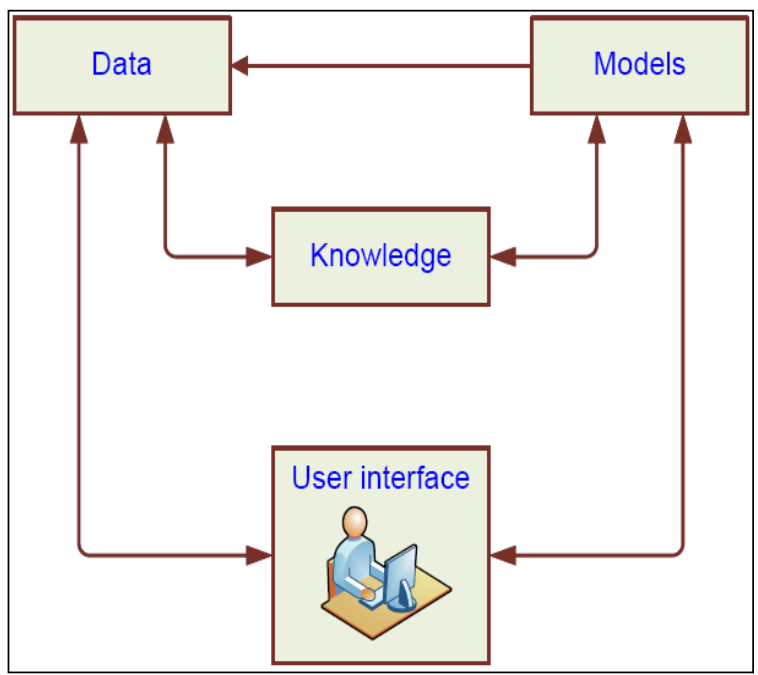

Fig. 2. Components of DSS [2]

\section{B. Data Mining Approaches}

Data Mining, which also known as Knowledge Discovery in Databases (KDD), refers to the significant extraction of hidden, previously unknown and potentially useful information from data in databases. While DM and knowledge discovery in databases (or KDD) are frequently treated as synonyms, DM is actually part of the knowledge discovery process [5, 8]. Furthermore, Abdullah et. Al described the DM concept in connection with decision support systems (DSS), in decision support management terminology, DM can be consider as a decision support process in which decision maker is searching to generate rule for the help in decision making process $[9,7$, 16]. There are several other fields where data mining techniques has been applied by researchers such as, medical database and ERP database [1, 14].
Mainly, DM tasks has been divided into descriptive and predictive methods. Classification, clustering and rule association mining are most common techniques use for predictive and descriptive analysis [10]. Therefore, mainly scholars describe DM in three major tasks. As Zaine [5] stated in his book chapter about major techniques of DM as follows:

a) Classification: Classification analysis is the organization of data in given classes. Also known as supervised classification, the classification uses given class labels to order the objects in the data collection

Classification consider as an important task of DM. Using this approach data must be already defined a class label (target) attribute. Firstly we divide the classified data into two sets; training and testing data [11]. Where each datasets contains others atrributes also but one of the attributed must be defined as class lable attribute. Jiawei Han [11] described classification task in two steps process; first is model construction and the second is model usage. The main target of this task is to build the model by using training dataset and then assign unseen records into a class by using the trained model as accurately as possible. While training data set is use to build the model on the other hand testing data set is use to validate the model [10].

b) Clustering: Similar to classification, clustering is the organization of data in classes. However, unlike classification, in clustering, class labels are unknown and it is up to the clustering algorithm to discover acceptable classes. Clustering is also called unsupervised classification.

Clustering is one of the major task has been applying for DM, work on unsupervised data (no predefined classes) [12]. Clustering is a collection of data objects, clustered by taking similar object to one another within the same cluster, and dissimilar to the objects related in other clusters. Cluster differentiate by using similarities between data according to the characteristics found in the data and grouping similar data objects into clusters [11].

c) Association: Association analysis is the discovery of what are commonly called association rules. It studies the frequency of items occurring together in transactional databases, and based on a threshold called support, identifies the frequent item sets.

Data can be use to find association between several attributes, generate rules from data sets, this task is known as association rule mining [12]. Given a set of transactions, find rules that will predict the occurrence of an item based on the occurrences of other items in the transaction. The goal of association rule mining is to find all rules having support $\geq$ minsup (minimum support) threshold and confidence $\geq$ minconf (minimum confidence) threshold [10].

Moreover, association rule mining can be viewed as a twostep process, first, find all frequent itemsets: items satisfying minimum support. Second, generate strong association rules from the frequent itemsets: these rules must satisfy minimum support and minimum confidence [11].

\section{METHODOLOGY}


The main purpose of this paper is to discuss the enhancement of traditional customer's dealing process by using current technology. Figure.1 presented above describe the traditionally customer's dealing process. We presented the DSS model in this paper including DM abstract to generate new rules and patterns to maintain the KM. In order to reply on the customer requests, it will connect directly with KM which have managed all the previous given replies to the customers.

In addition, [15] presented the similar concept of combining the DSS and DM applications together to build joint application to solve evaluation and classification problem. Specially they presented hierarchical multi-attribute decision model which are generally use in decision analysis [17,18]. Basically, this concept attract our research presented in this paper where this research is not taking one techniques into consideration but we have designed a model which can work broadly with any kind of data mining in different phases of decision support system.

Therefore, this paper presents the extended model of the figure. 2 with the integration of DM abstract. The implementation of that model is from customer's point of view which also known as customer relationship management (CRM). The major purpose of the CRM is to build good relations with the customer in the form customer satisfaction and behavior. Moreover, CRM will provide the facility to understand the customer by evaluating customer behavior, profile, customer segmentation, loyalty and profitability [3].

\section{A. Explanation of the Proposed Model - (Figure-3)}

Proposed model in figure. 3 is the extended model of DSS components model (figure. 2) presented above. According to figure. 2 the broad view of four components in the DSS connected to fulfill the requirements of DSS. The components are; data, model, KM, and user interface. Our model in figure.3 is covering all the four major components of DSS with some integration of DM abstract and customer enquiry systems. Therefore, according to our proposed model we can divide them into four major parts again as per the requirements of DSS. The division is also described in table.1. The complete description of each part of the proposed model discussed in the subsections.

a) Part 1. Data: In the perspective of proposed model we are merging the DSS database with the DM abstraction. As DM tasks are more commonly use for data understanding and extraction new information from the data. In this scenario, firstly the queries will be asked from KM about suitable reply. If the decision is not available from $\mathrm{KM}$, then it will proceed to DM abstract through DSS interface with selected data required for DM process. Afterwards, we can suppose that selected data is enough to produce some new patterns and rules. Simultaneously, the extracted information and rules will be saved in KM. Moreover, the DSS part 1. (DM) has some more methods to find and generate more alternatives for selecting best solution. As selecting the optimal solution is the major concern of DSS.

b) Part 2. Models: This part of DSS is to maintain the models generated while looking for new solutions. The proposed model has presented another aspect of the model generation which are known as DM models. Whenever DM tasks such as; Classification, clustering and association applied on the data, the tasks need to first build the model for data understanding and train the data. In addition of DSS own models, DM models will also be work and save in KM. These model can be available for the same type of request in future correspondence with the customer.

TABLE I. Comparison Between DSS Model AND Proposed Model

[COMPILED BY AUTHOR]

\begin{tabular}{|c|c|c|}
\hline DSS Components & According to DSS Model (Figure. 2) & According to Proposed Model (Figure.3) \\
\hline $\begin{array}{c}\text { Part 1: } \\
\text { Data }\end{array}$ & Data related with DSS Database & DM Abstract; will provide more tasks to do on the saved \\
data
\end{tabular}

c) Part 3. Knowledge Management: This part considerably has more importance for improving customer's behaviour and satisfaction with fast process of customer enquiries and complaints. $\mathrm{KM}$ is one of the types of database but the major purpose of building $\mathrm{KM}$ is to provide the facility to reply directly on customer queries (in current scenario) by using experienced data without contacting with DSS database.
While replying on customer queries, the DM models, rules \& patterns, and DSS models will place in the KM for future correspondence with the customers. Those type of data placed in $\mathrm{KM}$ is also known as experienced data. As presented in the model firstly all customer's request are connected with the decision box. Whereas decision box search the decision using $\mathrm{KM}$ to find the proper answer from experienced data 
(knowledge base data). If the decision will not available through KM then let the query will drag towards the DSS interface. At last if DSS need more understanding and some other operations to apply on data then it will be forwarded to the DM abstract. DM cannot initiate the working on customer queries directly. According to the model, DM abstract will work only if DSS abstract will forward any query with suitable data to apply any of the DM task. The solution/models generated either by DSS abstract or DM abstract will be transmitted to KM. Finally, the KM is only channel from where the answer will be post back to the asked customer.

d) Part 4. User Interface: In the model the user interface delegated for receiving and sending the customer requests online. According to the scenario detailed in the model a customer is the general term we use in this paper. Whereas a customer may be a type of user, purchaser, seller, influencer, or enquirer etc. Therefore, the raised query may also have several type such as; suggestions, requisitions, questionnaires, sales enquiries, and reclamations depend on the customer type. The major task of this model is to improve customer's satisfaction and behavior. Therefore, we need to build a interface which can support both DSS and DM abstracts. Finally, both abstract must connect with same KM.

\section{CONCLUSION AND FUTURE WORK}

In conclusion, we proposed the extended version of the DSS model with new perspective of DM and KM. Working on customer's query using DM and DSS abstract may work better than before. The concept of the model can also help to increase customer's satisfaction, behavior and ultimately the growth of the organization. As customer's plays significant role for making organization good will in the market. Therefore, the model may provide help for dealing with the customers in more efficient manner.

In future, the practical implementation of the proposed model with different attributed data may provide some fruitful results. Actually, each DM tasks deal with special attributed data. Model Implementation on real world data will guide more about the applicability of the model. Our future concerns is to apply this model using medical or student data for taking decision on student/patient online queries.

\section{Reference}

[1] Abdullah Saad ALMALAISE ALGHAMDI, "Efficient Implementation of FP Growth Algorithm Data Mining on Medical Data", International Journal of Computer Science and Network Security (IJCSNS)-2011.

[2] Efraim Turban, Ramesh Sharda, Dursun Delen, Decision Support and Business Intelligence, 9th Edition, published by Pearson Education, Prentice Hall-2011.

[3] Ruey-Shun Chen, Ruey-Chyi Wu and J. Y. Chen, "Data Mining Application in Customer Relationship Management of Credit Card Business", Institute of Information Management,Taiwan.

[4] Hai Wang, Shouhong Wang, Medical Knowledge Acquisition through Data Mining, Proceedings of 2008 IEEE International Symposium on IT in Medicine and Education.

[5] Osmar R. Zaïane, "Chapter I: Introduction to Data Mining", CMPUT690 Principles of Knowledge Discovery in Databases, 1999.

[6] Abdullah Al- Mudimigh, Farrukh Saleem, Zahid Ullah, The Effects Of Data Mining In ERP-CRM Model - A Case Study Of MADAR, WSEAS Transaction, 2009

[7] Dr. Abdullah Al- Mudimigh, Farrukh Saleem, Zahid Ullah, The Role Of Data Mining In ERP-CRM Model", International Conference on

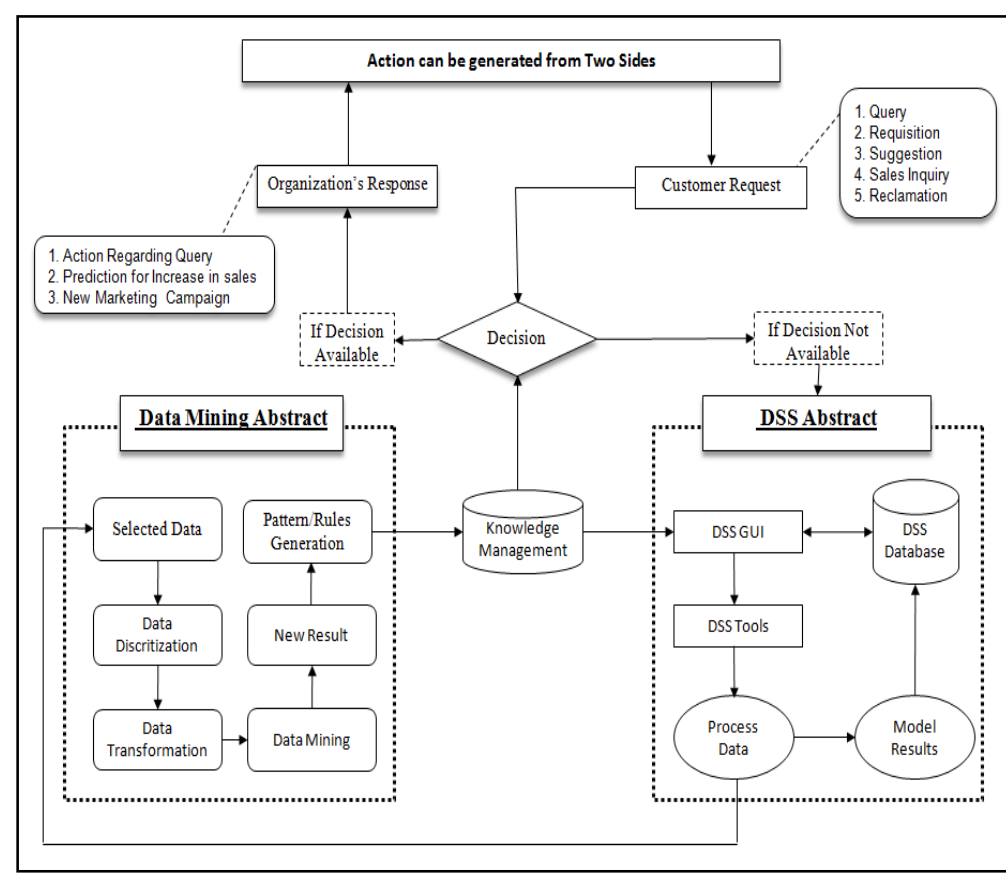

Fig. 3. Integrated Model of DSS with Data Mining Abstract [Proposed by Author]

Applied Computer \& Applied Computational Science (ACACOS '09), Hangzhou, China, 2009.

[8] Abdullah s. Al- Mudimigh, Farrukh Saleem, Zahid Ullah, Fahad N. AlAboud, Implementation of Data Mining Engine on CRM -Improve Customer Satisfaction", IEEE / ICICT-2009, Third International Conference on Information \& Communication Technologies, Karachi, Pakistan, 2009.

[9] Dr. Abdullah Al- Mudimigh, Farrukh Saleem, Zahid Ullah, Efficient Implementation of Data Mining: Improve Customer's Behavior", The 7th IEEE/ACS, International Conference on Computer Systems and Applications, Rabat, Morocco, to be held on May, 10-13, 2009.

[10] Pang-Ning Tan, Michael Steinbach \& Vipin Kumar, "Introduction to Data Mining", Addison Wesley, 2005, ISBN 0321321367.

[11] Jiawei Han, Micheline Kamber, Jian Pei, "Data Mining: Concepts and Techniques", 2nd edition, 2005, Morgan Kaufmann, ISBN 1558609016

[12] Farrukh Saleem, Areej Malibari, Data Mining Course In Information System Department-Case Study Of King Abdulaziz University, Ieee, ICEED- 3rd International Congress on Engineering Education, 7th - 8th December, 2011.

[13] S.C. Hui, G. Jha, "Data Mining for Customer Service Support", Information \& Management, Elsevier 2000.

[14] Abdullah Saad Almalaise Alghamdi, "Rules Generation from ERP Database: A Successful Implementation of Data Mining", IJCSNS International Journal of Computer Science and Network Security, VOL.12 No.3, March 2012.

[15] Marko Bohanec, Blaž Zupan, Integrating Decision Support and Data Mining by Hierarchical Multi-Attribute DecisionModels, University of Ljubljana, Slovenia, Baylor College of Medicine, Houston, U.S.A.

[16] Farrukh Saleem, Abdullah Saad Almalaise Alghamdi, "Implementation of data mining approach for building automated decision support systems", Information Society (i-Society), 2012 International Conference, London, UK, pp. 127 - 130, June 2012.

[17] Angehm, A.A.: Supporting multi-criteria decision making. In: Holtham C. (ed.): Execu-tive Information

[18] Systems and Decision Support. Chapman \& Hall (1992) Clemen, R.T.: Making Hard Decisions: An Introduction to Decision Analysis. Duxbury Press (1996) 